\title{
Painlevé Property of Monodromy Preserving Deformation Equations and the Analyticity of $\tau$ Functions
}

\author{
By \\ Tetsuji Mrws*
}

\section{§1. Introduction}

In this paper we shall prove the following two conjectures posed in [1] by using the Clifford operator method developed in [2].

Conjecture 1. The singularities of solutions to the monodromy preserving deformation equations are poles except for the fixed singularities.

Conjecture 2. The $\tau$ function is holomorphic except at the fixed singularities.

Painlevé's six equations were discovered in his classification of second order ordinary differential equations whose general solutions have no movable singularities other than poles. We call this property the Painleve property. It is now well-known [3], [4], [5] that all the Painlevé equations are obtained as monodromy preserving deformation equations. Thus in [1] the authors naturally conjectured that the general monodromy preserving deformation equations have the Painlevé property.

Ablowitz, Ramani and Segur [6] posed a similar conjecture: Every non linear ordinary differential equation obtained by an exact reduction of a non linear partial differential equation of inverse scattering class enjoy the Painlevé property. If we understand the meaning of an exact reduction as the reduction of a spectrum preserving deformation equation to a monodromy preserving deformation equation [5], their conjecture follows from our Theorem 1 below.

They also showed in some special examples that the Painlevé property can be proved by exploiting the Fredholm determinant. Oishi [7] obtained similar

Received November 12, 1980.

* Research Institute for Mathematical Sciences, Kyoto University Kyoto 606, Japan. 
results starting from bilinear differential forms of Painlevé equations. The use of the Fredholm theory in the monodromy problem originated in Hilbert [8]. We refer the reader to an excellent review by Saito [9]. Following Hilbert, Plemelj solved Riemann's problem [10]. Later Birkhoff [11] generalized the problem to the irregular singular case and solved it by a similar method.

In our theory of Clifford operators a Fredholm equation appears as the product formula [12]: The correlation function $\left\langle\varphi_{1}^{(1)} \cdots \varphi_{m}^{(n)}\right\rangle$ and the wave function $\left\langle\psi_{\alpha}^{*}\left(x_{0}\right) \varphi_{1}^{(1)} \cdots \varphi_{m}^{(n)} \psi_{\beta}(x)\right\rangle$ are the Fredholm determinant and the Fredholm minor of a certain integral equation which describes the quadratic kernel of the product operator $\varphi_{1}^{(1) \cdots} \varphi_{m}^{(n)}$. Unfortunately, in general, the usual Fredholm theory is not applicable because of the infinite length of contours for integrations. We avoid this difficulty by the following trick: Double the singularities so that we can use only finite contours (The twin problem). Then wipe out the irrelevant singularities by using Hilbert's boundary value problem. We refer the reader to the work of Ueno [13] which was very suggestive in thinking of the latter trick.

The notion of $\tau$ function has its origin in some exactly soluble models in theoretical physics [14], [15], [16]. In these models the correlation functions are expressible in terms of solutions to monodromy preserving deformation equations.

Okamoto [4] discovered that the logarithmic derivatives of 2 point correlation functions in these models serve as Hamiltonians when one writes the corresponding Painlevé equations in Hamiltonian forms. Inspired by this work Jimbo, Miwa and Ueno [1] introduced the notion of $\tau$ function for the general monodromy preserving deformation equations.

The analyticity of $\tau$ functions was proved by Okamoto [4] in the case of the six Painlevé equations. His method was to use the pole expansion of solutions at movable poles. If one knows the equations for $\tau$ functions it is rather easy to show the analyticity of $\tau$ functions [5]. But for the general monodromy preserving deformation equations neither the pole expansions nor the equations for $\tau$ functions are known. Fortunately, the analyticity of the $\tau$ functions for twin problems is a simple consequence of the Fredholm theory. Then we can prove the general case by collecting the irrelevant singularities into a single point.

The author thanks to M. Sato, K. Aomoto, K. Okamoto, M. Jimbo and 
K. Ueno for many helpful discussions.

\section{§2. Theorems}

We shall start with the statement of the problem.

Let $a_{1}, \ldots, a_{n}$ be distinct points in $\mathbb{C}$. We fix another point $x_{0} \in \mathbb{P}^{1}$ $-\left\{a_{1}, \ldots, a_{n}\right\}$. For each $a_{\mu}$ we attach a nonnegative integer $r_{\mu}$, which we call the rank of irregularity at $a_{\mu}$. We choose $r_{\mu}+1$ diagonal matrices

$$
T_{-j, \alpha}^{(\mu)}=\left(t_{-j, \alpha}^{(\mu)} \delta_{\alpha \beta}\right)_{\alpha, \beta=1, . ., m} \quad\left(j=1, \ldots, r_{\mu}\right) .
$$

We set

$$
T^{(\mu)}(x)=\sum_{j=1}^{r_{\mu}} T_{-j}^{(\mu)} \frac{\left(x-a_{\mu}\right)^{-j}}{-j}+T_{0}^{(\mu)} \log \left(x-a_{\mu}\right) .
$$

If $r_{\mu} \geqq 1$, we assume that

$$
t_{-r_{\mu}, \alpha}^{(\mu)} \neq t_{-r_{\mu}, \beta}^{(\mu)} \quad(\alpha \neq \beta)
$$

We also assume that

$$
\sum_{\mu=1}^{n} \sum_{\alpha=1}^{m} t_{0, \alpha}^{(\mu)}=0
$$

We call $a_{\mu}(\mu=1, \ldots, n)$ and $t_{-j, \alpha}^{(\mu)}\left(\mu=1, \ldots, n ; j=1, \ldots, r_{\mu} ; \alpha=1, \ldots, m\right)$ the deformation parameters. The parameters $t_{0, \alpha}^{(\mu)}(\alpha=1, \ldots, m)$ are called the exponents of formal monodromy at $a_{\mu}$. We set

$$
e_{\alpha}^{(\mu)}(x)=\exp \left\{\sum_{j=1}^{r_{\mu}} t_{-j, \alpha}^{(\mu)} \frac{\left(x-a_{\mu}\right)^{-j}}{-j}+t_{0, \alpha}^{(\mu)} \log \left(x-a_{\mu}\right)\right\}
$$

and

$$
\operatorname{Re}_{\alpha \beta}^{(\mu)}(x)=\operatorname{Re}\left(t_{-r_{\mu}, \alpha}^{(\mu)} \frac{\left(x-a_{\mu}\right)^{-r_{\mu}}}{-r_{\mu}}-t_{-r_{\mu}, \beta}^{(\mu)} \frac{\left(x-a_{\mu}\right)^{-r_{\mu}}}{-r_{\mu}}\right) .
$$

We choose a path $\gamma_{\mu}$ in $P^{1}-\left\{a_{1}, \ldots, a_{n}\right\}$ connecting $a_{\mu}$ to $x_{0}$ and an invertible $m \times m$ matrix $C^{(\mu)}$ which we call the connection matrix from $a_{\mu}$ to $x_{0}$. We assume that $\gamma_{1}, \ldots, \gamma_{n}$ are disjoint and located anticlockwise in this order.

If $r_{\mu} \geqq 1$ we define sectors $\mathscr{S}_{\rho \delta}^{(\mu l)}$ at $a_{\mu}$ :

$$
\begin{aligned}
\mathscr{S}_{\rho \delta}^{(\mu l)}=\{x \in \boldsymbol{C} \mid 0 & <\left|x-a_{\mu}\right|<\delta, \\
& \left.\rho+\pi \frac{l-1}{r_{\mu}}-\delta<\arg \left(x-a_{\mu}\right)<\rho+\pi \frac{l}{r_{\mu}}\right\} .
\end{aligned}
$$


We assume that $\gamma_{\mu}$ flows into $a_{\mu}$ through $\mathscr{S}_{\rho \delta}^{(\mu l)}$. For the moment we fix $\rho$ and choose $\delta$ small enough so that

$$
\operatorname{Re}_{\alpha \beta}^{(\mu)}(x) \neq 0(\alpha \neq \beta) \quad \text { for } \quad x \in \mathscr{S}_{\rho \delta}^{(\mu l)} \cap \mathscr{S}_{\rho \delta}^{(\mu l+1)} .
$$

We choose $2 r_{\mu} m \times m$ nilpotent matrices

$$
\Lambda^{(\mu l)}=\left(\lambda_{\alpha \beta}^{(\mu l)}\right)_{\alpha, \beta=1, \ldots, m} \quad\left(l=1, \ldots, 2 r_{\mu}\right),
$$

such that

$$
\lambda_{\alpha \beta}^{(\mu l)}=0 \quad \text { if } \quad \operatorname{Re}_{\alpha \beta}^{(\mu)}(x) \geqq 0 \quad \text { for } \quad x \in \mathscr{S}_{\rho \delta}^{(\mu l)} \cap \mathscr{S}_{\rho \delta}^{(\mu l+1)}
$$

We set

$$
\begin{aligned}
& S_{l}^{(\mu)}=\left(1+\Lambda_{l}^{(\mu)}\right)^{-1}, \\
& M^{(\mu)}=C^{(\mu)-1} e^{2 \pi i T_{0}^{(\mu)}} S_{2 r_{\mu}}^{(\mu)-1} \cdots S_{1}^{(\mu)-1} C^{(\mu)} .
\end{aligned}
$$

If $r_{\mu}=0$ we set

$$
M^{(\mu)}=C^{(\mu)-1} e^{2 \pi i T_{0}^{(\mu)}} C^{(\mu)} .
$$

$S_{l}^{(\mu)}$ is called a Stokes multiplier and $M^{(\mu)}$ is called a monodromy matrix. The consistency of monodromy reads as

$$
M^{(n)} \cdots M^{(1)}=1 .
$$

The Riemann's monodromy problem is to find an $m \times m$ matrix $Y(x)$ which satisfies the following:

(M1) $Y\left(x_{0}\right)=1$.

(M2) $Y(x)$ can be analytically continued in the universal covering of $\mathbb{P}^{1}-\left\{a_{1}, \ldots, a_{n}\right\}$.

(M3) The analytic continuation of $Y(x)$ along $\gamma_{\mu}$ is of the form: If $r_{\mu}=0$,

$$
Y(x)=G^{(\mu)} \hat{Y}^{(\mu)}(x)\left(x-a_{\mu}\right)^{T_{0}^{(\mu)}} C^{(\mu)}
$$

and if $r_{\mu} \geqq 1$,

$$
Y(x) \sim G^{(\mu)} \hat{Y}^{(\mu)}(x) e^{T^{(\mu)}(x)} S_{l-1}^{(\mu)-1} \cdots S_{1}^{(\mu)-1} C^{(\mu)}
$$

in $\mathscr{S}_{l \delta}^{(\mu l)}\left(l=1, \ldots, 2 r_{\mu}+1\right)$. Here $G^{(\mu)}$ is an invertible matrix independent of $x$ and

$$
\hat{Y}^{(\mu)}(x)=1+\sum_{j=1}^{\infty} Y_{j}^{(\mu)}\left(x-a_{\mu}\right)^{j}
$$

is a convergent (if $r_{\mu}=0$ ) or a formal (if $r_{\mu} \geqq 1$ ) power series. 
Let us denote by $D^{(\mu)}(x)$ the diagonal part of $\hat{Y}^{(\mu)}(x)$ and set

$$
F^{(\mu)}(x)=\hat{Y}^{(\mu)}(x) D^{(\mu)}(x)^{-1}=\sum_{j=0}^{\infty} F_{j}^{(\mu)}\left(x-a_{\mu}\right)^{j} \quad\left(F_{0}^{(\mu)}=1\right) .
$$

Then $F_{j}^{(\mu)}(j \geqq 1)$ is diagonal free.

For a given set of matrices $G^{(\mu)}$ and $F_{j}^{(\mu)}\left(\mu=1, \ldots, n ; j=1, \ldots, r_{\mu}\right)$ we define a rational one form $A(x) d x$ as follows: The poles of $A(x) d x$ are $a_{1}, \ldots, a_{n}$. The singular part of $A(x) d x$ at $a_{\mu}$ is determined by

$$
\begin{aligned}
\Lambda(x) d x \equiv & G^{(\mu)}\left(\sum_{j=0}^{r_{\mu}} F_{j}^{(\mu)}\left(x-a_{\mu}\right)^{j}\right) \cdot\left(\sum_{j=0}^{r_{\mu}} T_{-j}^{(\mu)}\left(x-a_{\mu}\right)^{-j-1} d x\right) \\
& \times\left(\sum_{j=0}^{r_{\mu}} F_{j}^{(\mu)}\left(x-a_{\mu}\right)^{j}\right)^{-1} G^{(\mu)-1} \bmod \left(x-a_{\mu}\right)^{0} .
\end{aligned}
$$

If an $m \times m$ matrix $Y(x)$ satisfying the monodromy property (M1)-(M3) ever exists, it is unique and solves the following linear ordinary differential equation:

$$
\frac{d Y(x)}{d x}=A(x) Y(x)
$$

The rational coefficient $A(x)$ is determined by (2.19) and satisfies

$$
\sum_{\mu=1}^{n} \operatorname{Res}_{x=a_{\mu}} A(x) d x=0 .
$$

Conversely, let us assume that $A(x)$, given by (2.19), satisfies (2.21). We also assume that

$$
t_{0, \alpha}^{(\mu)} \not \equiv t_{0, \beta}^{(\mu)} \quad \bmod \mathbb{Z} \quad \text { if } \quad r_{\mu}=0 .
$$

Then the solution to (2.20), determined by (M1), enjoys (M2) and (M3). Now we fix the reference point $x_{0}$ and the argument $\rho$ and vary the paths $\gamma_{1}, \ldots, \gamma_{n}$ continuously. We assume that

$$
\arg \left(t_{-r_{\mu}, \alpha}^{(\mu)}-t_{-r_{\mu}, \beta}^{(\mu)}\right)-r_{\mu} \rho \not \equiv \frac{\pi}{2} \quad \bmod \pi \mathbb{Z} \quad(1 \leqq \mu \leqq n ; 1 \leqq \alpha \neq \beta \leqq m) .
$$

The correspondence from $\left(a_{\mu}, t_{-j, \alpha}^{(\mu)}, t_{0, \alpha}^{(\mu)}, G_{\alpha \beta}^{(\mu)}, F_{j \alpha \beta}^{(\mu)}\right)_{1 \leqq \mu \leqq n, 1 \leqq j \leqq r_{\mu}, 1 \leqq \alpha . \beta \leqq m}$ to $\left(a_{\mu}, t_{-j, \alpha}^{(\mu)}, t_{0, \alpha}^{(\mu)}, C_{\sigma \beta}^{(\mu)}, \lambda_{\alpha \beta}^{(\mu l)}\right)_{1 \leqq \mu \leqq n, 1 \leqq j \leqq r_{\mu}, 1 \leqq \alpha, \beta \leqq m .1 \leqq l \leqq 2 r_{\mu}}$ is locally biholomorphic.

We denote by $\mathscr{T}$ the universal covering of the space of deformation parameters:

$$
\begin{aligned}
\left\{\left(a_{\mu}, t_{-j, \alpha}^{(\mu)}\right)_{1 \leqq \mu \leqq n, 1 \leqq j \leqq r_{k}, 1 \leqq \alpha \leqq m} \in C^{n+m \Sigma_{\mu=1}^{n} r_{\mu} \mid a_{\mu}} \neq\right. & \neq a_{v}(\mu \neq v), \\
t_{-r_{\mu}, \alpha}^{(\mu)} & \left.\neq t_{-r_{\mu}, \beta}^{(\mu)}(\alpha \neq \beta)\right\} .
\end{aligned}
$$


The exceptional hyperplanes $\left\{a_{\mu}-a_{v}=0\right\}(1 \leqq \mu \neq v \leqq n)$ and $\left\{t_{-r_{\mu}, \alpha}^{(\mu)}-t_{-r_{\mu}, \beta}^{(\mu)}=0\right\}$ $(1 \leqq \mu \leqq n, 1 \leqq \alpha \neq \beta \leqq m)$ are called the fixed singularities. We denote by $\mathscr{N}$ the fiber space over $\mathscr{T}$ whose fiber is the space of singularity data $\left(t_{0, \alpha}^{(\mu)}, G_{\alpha \beta}^{(\mu)}\right.$, $\left.F_{j, \alpha}^{(\mu)}\right)_{1 \leqq \mu \leqq n, 1 \leqq \alpha, \beta \leqq m, 1 \leqq j \leqq r_{\mu}}$ satisfying (2.4), (2.21) and (2.22). We denote by $\mathscr{N}_{\rho}$ the subspace of $\mathscr{N}$ subject to the condition (2.23).

We construct another fiber space $\mathscr{M}$ over $\mathscr{T}$. We denote by $\mathscr{H}_{\rho}$ the fiber space over $\mathscr{T}$ whose fiber is the space of monodromy data $\left(t_{0, \alpha}^{(\mu)}, C_{\alpha \beta}^{(\mu)}, \lambda_{\alpha \beta}^{(\mu l)}\right)_{1 \leqq \mu \leqq n}$, $1 \leqq \alpha . \beta \leqq m, 1 \leqq l \leqq 2 r_{\mu}$ satisfying (2.4), (2.10) and (2.14). We fix $x_{0}=\infty$. We also choose the paths $\gamma_{1}, \ldots, \gamma_{n}$ so that they are continuously varying. Then by solving the system (2.20) we define a holomorphic map from each connected component of $\mathscr{N}_{\rho}$ to $\mathscr{M}_{\rho}$. We define $\mathscr{M}$ by patching these holomorphic maps (with different $\rho$ 's and for different components).

We denote by $\mathscr{R}$ the holomorphic map from $\mathscr{N}$ to $\mathscr{M}$ thus obtained. $\mathscr{R}$ is one to one and locally biholomorphic. In Section 3 we shall prove

Theorem 1. The inverse of $\mathscr{R}$ is meromorphic.

This is equivalent to say that $Y(x)$ is meromorphic on $\mathscr{l l}$.

It is known [1] that a horizontal leaf in $\mathscr{M}$ represents a solution to the monodromy preserving deformation equation, if the former has a non void intersection with the image of $\mathscr{N}$, and vice versa. Thus Theorem 1 asserts that the singularities of a solution to the monodromy preserving deformation equation are poles except for fixed singularities.

Let us denote by $d_{t}$ the exterior differentiation on $\mathscr{T}$. We define a 1-form on $\mathscr{M}$ by

$$
\omega=-\sum_{\mu=1}^{n} \operatorname{Res}_{x=a_{\mu}} \hat{Y}^{(\mu)}(x)^{-1} \frac{d \hat{Y}^{(\mu)}(x)}{d x} d x d_{t} T^{(\mu)}(x) .
$$

It is known [1] that $\omega$ is $d_{t}$-closed. Theorem 1 asserts that $\omega$ is meromorphic. In Section 4 we shall prove

Theorem 2. The poles of $\omega$ are simple and the residues are positive integers.

We define $\tau$ on each horizontal leaf in $\mathscr{M}$ by

$$
d_{t} \log \tau=\omega .
$$

Then Theorem 2 asserts that $\tau$ is holomorphic. 


\section{§3. Proof of Theorem $\mathbb{1}$}

In [2] we have shown that the solution $Y(x)$ to the monodromy problem for small parameters $t_{-j, \alpha}^{(\mu)}, \lambda_{\alpha \beta}^{(\mu l)}\left(1 \leqq \mu \leqq n, 0 \leqq j \leqq r_{\mu}, 1 \leqq \alpha, \beta \leqq m, 1 \leqq l \leqq 2 r_{\mu}\right)$ is given by the following expressions in terms of free fermion fields $\psi_{\alpha}^{*}(x), \psi_{\alpha}(x)$ and certain Clifford operators $\varphi_{\alpha}^{(\mu)}$ :

$$
\left.Y(x)_{\alpha \beta}=2 \pi i\left(. x-x_{0}\right) \frac{\left\langle\psi_{x}^{*}\left(x_{0}\right) \varphi_{1}^{(1)} \cdots \varphi_{m}^{(n)}\right.}{\left\langle\varphi_{1}^{(1)} \cdots \varphi_{m}^{(n)}\right\rangle}\right\rangle
$$

In fact, applying the Wick's theorem to the right hand side we obtain a Neumann series for $Y(x)_{\alpha \beta}$. Then we can verify the monodromy property. The Neumann series does not converge for general values of parameters. This difficulty is not merely a technical one because the solution should have poles at certain values of the deformation parameters.

A more natural approach to give a precise meaning to (3.1) is to apply the Wick's theorem to $\left\langle\varphi_{1}^{(1)} \cdots \varphi_{m}^{(n)}\right\rangle$ and $\left\langle\psi_{\alpha}^{*}\left(x_{0}\right) \varphi_{1}^{(1)} \cdots \varphi_{m}^{(n)} \psi_{\beta}(x)\right\rangle$ separately, and to express $Y(x)_{\alpha \beta}$ as a quotient of two holomorphic functions. For example, with an appropriate choice of integration contours we obtain the following expression for the correlation function $\left\langle\varphi_{1}^{(1)} \cdots \varphi_{m}^{(n)}\right\rangle$ :

$$
\begin{aligned}
& \left\langle\varphi_{1}^{(1)} \cdots \varphi_{m}^{(n)}\right\rangle=\sum_{l=0}^{\infty} \frac{(-)^{l}}{l !} \sum_{\mu_{1}, \ldots, \mu_{l}=1}^{n} \sum_{\alpha_{1}, \ldots, \alpha_{l}=1}^{m} \int d x_{1} \cdots \int d x_{l} \times \\
& \quad \times \operatorname{det}\left(-\frac{1}{2 \pi i}-\frac{1}{x_{j}-x_{k}} K_{\alpha_{j} \alpha_{k}}^{\left(u_{j} u_{k}\right)} \frac{e_{\alpha_{j}}^{\left(\mu_{J}\right)}\left(x_{k}\right)}{e_{\alpha_{k}}^{\left(\mu_{k}\right)}\left(x_{k}\right)}\right)_{j, k=1, \ldots, l} .
\end{aligned}
$$

Unfortunately, the usual Fredholm theory does not work because the contours given in [2] are of infinite length.

To overcome this difficulty we recall our previous results [16], [17] on some physical models. The contours employed there flow into certain sectors where the integrand is exponentially decreasing otherwise they are compact. Hence in these models the analyticity of the correlation functions (=the $\tau$ functions) are obvious.

If we treat general monodromy data we cannot expect to use such good contours. We exploit the following tricks instead:

i) Doubling of singularities.

ii) Hilbert's boundary value problem.

Namely, we first solve a monodromy problem with doubled singularity. We 
call it the twin problem. The doubling enables us to use good contours in the above sense. Then we solve a boundary value problem in the sense of Hilbert and Plemelj [8], [10] to wipe out the irrelevant singularities.

Let us consider twins of deformation parameters $\left(a_{\mu}, t_{-j, \alpha}^{(\mu)}\right)_{1 \leqq j \leqq r_{\mu}, 1 \leqq \alpha \leqq m}$ and $\left(b_{\mu}, s_{-j, \alpha}^{(\mu)}\right)_{1 \leqq j \leqq r_{\mu}, 1 \leqq \alpha \leqq m}$. We assume that $a_{1}, \ldots, a_{n}, b_{1}, \ldots, b_{n}$ are distinct and $t_{-r_{\mu}, \alpha}^{(\mu)} \neq t_{-r_{\mu}, \beta}^{(\mu)}, s_{-r_{\mu}, \alpha}^{(\mu)} \neq s_{-r_{\mu}, \beta}^{(\mu)}(\alpha \neq \beta)$. We also assume that

$$
\left|t_{-r_{\mu}, \alpha}^{(\mu)}-s_{-r_{\mu}, \alpha}^{(\mu)}\right| \ll 1
$$

For each $(\mu l)$ we can choose a permutation $\sigma^{(\mu l)} \in \mathfrak{S}_{m}$ so that

$$
\operatorname{Re}_{\alpha \beta}^{(\mu)}(x)<0 \text { for } x \text { s.t. } \arg \left(x-a_{\mu}\right)=\rho+\frac{\pi}{r_{\mu}}(l-1)
$$

if and only if $\sigma^{(\mu l)}(\alpha)>\sigma^{(\mu l)}(\beta)$.

Taking a small positive constant $\varepsilon$ we set

$$
\begin{aligned}
\gamma_{k}^{(\mu l)} & =\left\{x \in \boldsymbol{C}|0<| x-a_{\mu} \mid \ll 1, \arg \left(x-a_{\mu}\right)=\rho+\frac{\pi}{r_{\mu}}(l-1)+k \varepsilon\right\}, \\
\gamma_{k}^{(\mu l) \prime} & =\left\{x \in \boldsymbol{C}|0<| x-b_{\mu} \mid \ll 1, \arg \left(x-b_{\mu}\right)=\rho-\frac{\pi}{r_{\mu}} l-k \varepsilon\right\} .
\end{aligned}
$$

We choose $n$ distinct paths $\Gamma\left(a_{\mu}, b_{u}\right)(\mu=1, \ldots, n)$ which comes from $b_{\mu}$ and flows into $a_{\mu}$. We fix contours $I^{(\mu)}$ and $I I_{k}^{(\mu l)}(1 \leqq k \leqq m-1)$ as follows. $I^{(\mu)}$ is a closed path surrounding $\Gamma\left(a_{\mu}, b_{\mu}\right)$ anticlockwise. We take $I^{(\mu)}(\mu=1, \ldots, n)$ so that the regions $\mathscr{S}^{(\mu)}$ surrounded by $I^{(\mu)}$ are disjoint. $I I_{k}^{(\mu l)}$ is a path in $\mathscr{S}^{(\mu)}$ which connects $\gamma_{k}^{(\mu l)}$ and $\gamma_{k}^{(\mu l) \prime}$ in the same direction with $\Gamma\left(a_{\mu}, b_{\mu}\right)$. They should be disjoint and located anticlockwise (resp. clockwise), viewed from $a_{\mu}$ (resp. $b_{\mu}$ ), in the order $I I_{1}^{(\mu 1)}, I I_{2}^{(\mu 1)}, \ldots, I I_{m-1}^{(\mu 1)}, I I_{1}^{(\mu 2)}, \ldots, I I_{m-1}^{\left(\mu 2 r_{\mu}\right)}$. If we ignore the fine structure $I I_{1}^{(\mu l)}, \ldots, I I_{m-1}^{(\mu l)}$, we write simply $I I^{(\mu l)}$. The contours $I^{(\mu)}$ 's and $I I^{(\mu l)}$ 's divide $\boldsymbol{P}$ into $1+\sum_{\mu=1}^{n} \max \left(1,2 r_{\mu}\right)$ pieces. We denote by $\mathscr{S}^{(0)}$ the region containing $\infty$. We denote by $\mathscr{S}^{(\mu l)}$ the region containing the segments $\left\{x \in \boldsymbol{C}|0<| x-a_{\mu} \mid \ll 1, \arg \left(x-a_{\mu}\right)=p+\pi(l-1 / 2) / r_{\mu}\right\}$ and $\left\{x \in \boldsymbol{C}|0<| x-b_{\mu} \mid \ll 1\right.$, $\left.\arg \left(x-b_{\mu}\right)=p-\pi(l+1 / 2) / r_{\mu}\right\}$.

We choose a point $x_{0}$ in $\mathscr{S}^{(0)}$. We set

$$
\begin{aligned}
& \varepsilon_{\alpha}^{(\mu)}(x) \\
& =\frac{\exp \left\{\sum_{j=1}^{r_{\mu}} t_{-j, \alpha}^{(\mu)} \frac{\left(x-a_{\mu}\right)^{-j}}{-j}+t_{0, \alpha}^{(\mu)} \log \left(x-a_{\mu}\right)\right\}}{\exp \left\{\sum_{j=1}^{r_{\mu}} s_{-j, \alpha}^{(\mu)} \frac{\left(x-b_{\mu}\right)^{-j}}{-j}+t_{0, \alpha}^{(\mu)} \log \left(x-b_{\mu}\right)\right\}} .
\end{aligned}
$$


Now we consider the following monodromy problem.

The twin problem: Find an $m \times m$ matrix $Z(x)$ such that

(T1) $Z\left(x_{0}\right)=1$,

(T2) $Z(x)$ is single-valued holomorphic in $\mathscr{S}^{(0)}$,

(T3) $Z(x)$ can be continued holomorphically to $\mathscr{P}^{(\mu 1)}, \ldots, \mathscr{P}^{\left(\mu 2 r_{\mu}+1\right)}$, successively, and in $\mathscr{P}^{(\mu l)}$, when $\left|x-a_{\mu}\right| \rightarrow 0$ (as well as $\left|x-b_{\mu}\right| \rightarrow 0$ ) it has an asymptotic expansion, which is independent of $l$.

We shall solve the twin problem by exploiting Clifford operators. We use free fermion fields $\psi_{\alpha}(x), \psi_{\alpha}^{*}(x)\left(x \in \mathscr{S}^{(0)}\right), \phi_{\alpha}^{(\mu)}(x) \phi_{\alpha}^{*(\mu)}(x)\left(x \in I^{(\mu)}\right)$ and $\phi_{\alpha}^{(\mu l)}(x), \phi_{\alpha}^{*(\mu l)}(x)\left(x \in I I^{(\mu l)}\right)$. The expectation value is defined as follows:

$$
\left\langle\phi_{1}(x) \phi_{2}\left(x^{\prime}\right)\right\rangle=0,\left\langle\phi_{1}^{*}(x) \phi_{2}^{*}\left(x^{\prime}\right)\right\rangle=0
$$

and

$$
\left\langle\phi_{1}^{*}(x) \phi_{2}\left(x^{\prime}\right)\right\rangle=-\left\langle\phi_{2}\left(x^{\prime}\right) \phi_{1}^{*}(x)\right\rangle,
$$

if $\phi_{j}=\psi_{\alpha}, \phi_{\alpha}^{(\mu)}$ or $\phi_{\alpha}^{(\mu l)}$ and if $\phi_{j}^{*}=\psi_{\alpha}^{*}, \phi_{\alpha}^{*(\mu)}$ or $\phi_{\alpha}^{*(\mu l)}$.

$$
\begin{aligned}
& \left\langle\psi_{\alpha}^{*}(x) \psi_{\beta}\left(x^{\prime}\right)\right\rangle=\frac{1}{2 \pi} \frac{i}{x-x^{\prime}} \delta_{\alpha \beta}\left(x \neq x^{\prime}\right) . \\
& \left\langle\psi_{\alpha}^{*}(x) \phi_{\beta}^{(\mu)}\left(x^{\prime}\right)\right\rangle=\left(C^{(\mu)-1}\right)_{\alpha \beta} \frac{1}{2 \pi} \frac{i}{x-x^{\prime}} . \\
& \left\langle\phi_{\alpha}^{*(\mu)}(x) \psi_{\beta}\left(x^{\prime}\right)\right\rangle=C_{\alpha \beta}^{(\mu)} \frac{1}{2 \pi} \frac{i}{x-x^{\prime}} . \\
& \left\langle\psi_{\alpha}^{*}(x) \phi_{\beta}^{(\mu l)}\left(x^{\prime}\right)\right\rangle=\left\langle\phi_{\alpha}^{*(\mu l)}(x) \psi_{\beta}\left(x^{\prime}\right)\right\rangle=0 . \\
& \left\langle\phi_{\alpha}^{*(\mu)}(x) \phi_{\beta}^{(v)}\left(x^{\prime}\right)\right\rangle=\left(1-\delta_{\mu v}\right)\left(C^{(\mu)} C^{(v)-1}\right)_{\alpha \beta} \frac{1}{2 \pi} \frac{i}{x-x^{\prime}} . \\
& \left\langle\phi_{\alpha}^{*(\mu)}(x) \phi_{\beta}^{(v l)}\left(x^{\prime}\right)\right\rangle=\left\langle\phi_{\alpha}^{*(\mu l)}(x) \phi_{\beta}^{(v)}\left(x^{\prime}\right)\right\rangle=0 . \\
& \left\langle\phi_{\alpha}^{*(\mu l)}(x) \phi_{\beta}^{\left(v l^{\prime}\right)}\left(x^{\prime}\right)\right\rangle=\delta_{\mu \nu} \delta_{l l^{\prime}} \lambda_{\alpha \beta}^{(\mu l)} \delta\left(x-x^{\prime}\right) .
\end{aligned}
$$

We set

$$
\begin{aligned}
& \psi_{\alpha}^{(\mu)}(x)=\left\{\begin{array}{lll}
\phi_{\alpha}^{(\mu)}(x) & \text { if } & x \in I^{(\mu)} \\
\phi_{\alpha}^{(\mu l)}(x) & \text { if } & x \in I^{(\mu l)}
\end{array}\right. \\
& \psi_{\alpha}^{*(\mu)}(x)=\left\{\begin{array}{lll}
\phi_{\alpha}^{*(\mu)}(x) & \text { if } & x \in I^{(\mu)} \\
\phi_{\alpha}^{*(\mu l)}(x) & \text { if } & x \in I I^{(\mu l)}
\end{array}\right. \\
& R_{\alpha}^{(\mu)}\left(x, x^{\prime}\right)=\frac{1}{2 \pi i} \frac{1}{x-x^{\prime}} \frac{\varepsilon_{\alpha}^{(\mu)}\left(x^{\prime}\right)}{\varepsilon_{\alpha}^{(\mu)}(x)}
\end{aligned}
$$


and define a Clifford operator $\varphi_{\alpha}^{(\mu)}$ by

$$
\varphi_{\alpha}^{(\mu)}=\exp \rho_{\alpha}^{(\mu)}, \rho_{\alpha}^{(\mu)}=\iint d x d x^{\prime} R_{\alpha}^{(\mu)}\left(x, x^{\prime}\right) \psi_{\alpha}^{(\mu)}(x) \psi_{\alpha}^{*(\mu)}\left(x^{\prime}\right)
$$

In (3.18), if $x$ and $x^{\prime}$ belong to the same contour, we take $x^{\prime}$ to be on the right bank of the contour for $x$.

We shall construct the solution $Z(x)$ in the form

$$
Z(x)=2 \pi i\left(x-x_{0}\right) \frac{\left\langle\psi_{\alpha}^{*}\left(x_{0}\right) \varphi_{1}^{(1)} \cdots \varphi_{m}^{(n)} \psi_{\beta}(x)\right\rangle}{\left\langle\varphi_{1}^{(1)} \cdots \varphi_{m}^{(n)}\right\rangle} .
$$

Let us consider $\left\langle\varphi_{1}^{(1)} \cdots \varphi_{m}^{(n)}\right\rangle$ first. We set

$$
K_{a \beta}^{(\mu v)}(x, y)=\frac{1}{2 \pi i} \frac{1}{x-y} K_{\alpha \beta}^{(\mu v)}(y) \frac{\varepsilon_{\alpha}^{(\mu)}(y)}{\varepsilon_{\beta}^{(v)}(y)}
$$

where $K_{\alpha \beta}^{(\mu \nu)}(y)$ is defined on the contours as follows:

$$
\begin{aligned}
& K_{\alpha \beta}^{(\mu v)}(y) \\
& = \begin{cases}\left(1-\delta_{\mu v}\right)\left(C^{(\mu)} C^{(v)-1}\right)_{\alpha \beta} & \text { if } y \in I^{(v)} \\
\delta_{\mu \nu} \lambda_{\alpha \beta}^{(v l)} & \text { if } y \in I I_{\sigma^{(\nu l)}(\beta)}^{(v l)} \\
0 & \text { otherwise. }\end{cases}
\end{aligned}
$$

The Wick's theorem gives us the following expression for $\left\langle\varphi_{1}^{(1)} \cdots \varphi_{m}^{(n)}\right\rangle$ :

$$
\begin{aligned}
\left\langle\varphi_{1}^{(1)} \cdots \varphi_{m}^{(n)}\right\rangle= & \sum_{l=0}^{\infty} \frac{(-1)^{l}}{l !} \sum_{\mu_{1}, \ldots, \mu_{l}=1}^{n} \sum_{\alpha_{1}, \ldots, \alpha_{l}=1}^{m} \\
& \times \int d x_{1} \cdots \int d x_{l} \operatorname{det}\left(K_{\alpha_{j} \alpha_{k}}^{\left(\mu_{j} \mu_{k}\right)}\left(x_{j}, x_{k}\right)\right)_{j, k=1, \ldots, l} .
\end{aligned}
$$

Here the integration is over $\left(\cup_{\mu=1}^{n} I^{(\mu)}\right) \cup\left(\bigcup_{\substack{l=1, \ldots, 2 r_{\mu} \\ k=1, \ldots, m-1}} I I_{k}^{(\mu l)}\right)$. The Fredholm theory assures that $\left\langle\varphi_{1}^{(1)} \cdots \varphi_{m}^{(n)}\right\rangle$ is holomorphic with respect to the parameters $a_{\mu}, b_{\mu}, t_{-j, \alpha}^{(\mu)}, s_{-j, \alpha}^{(\mu)}, \lambda_{\alpha \beta}^{(\mu l)}$ and $C_{\alpha \beta}^{(\mu)}\left(1 \leqq \mu \leqq n, 0 \leqq j \leqq r_{\mu}, 1 \leqq \alpha, \beta \leqq m, 1 \leqq l \leqq 2 r_{\mu}\right)$.

Similarly, $\left\langle\psi_{\alpha}^{*}\left(x_{0}\right) \varphi_{1}^{(1)} \cdots \varphi_{m}^{(n)} \psi_{\beta}(x)\right\rangle$ is expressed as a Fredholm minor:

$$
\begin{aligned}
&=\sum_{l=0}^{\infty} \frac{(-)^{l}}{l !} \sum_{\mu_{1}, \ldots, \mu_{l}=1}^{n} \sum_{\alpha_{1}, \ldots, \alpha_{l}=1}^{m} \int d x_{1} \cdots \int d x_{l} \\
& \times \operatorname{det}\left[\begin{array}{cc}
\delta_{\alpha \beta} \frac{1}{2 \pi} \frac{i}{x_{0}-x} & \left(\left(C^{\left(\mu_{k}\right)-1}\right)_{\alpha \alpha_{k}} \frac{1}{2 \pi} \frac{i}{x_{0}-x_{k}} \varepsilon_{\alpha_{k}}^{\left(\mu_{k}\right)}\left(x_{k}\right)\right)_{k=1, \ldots, l} \\
\left(\frac{1}{2 \pi i} \frac{1}{x_{j}-x} \frac{1}{\varepsilon_{\alpha_{j}}^{\left(\mu_{j}\right)}\left(x_{j}\right)} C_{\alpha_{j} \beta}^{\left(\mu_{j}\right)}\right)_{j=1, \ldots, l} & \left(K_{\alpha_{j} \alpha_{k}}^{\left(\mu_{j} \mu_{k}\right)}\left(x_{j}, x_{k}\right)\right)_{j, k=1, \ldots, l}
\end{array}\right] .
\end{aligned}
$$


Applying the argument in the proof of Theorem 2.3 in [2] we can show that (3.19) with (3.22) and (3.23) gives us the solution to the twin problem, which is meromorphic with respect to the parameters.

For the moment we assume that

$$
\left\langle\varphi_{1}^{(1)} \cdots \varphi_{m}^{(n)}\right\rangle \neq 0 \text {. }
$$

We should wipe out irrelevant singularities $b_{1}, \ldots, b_{n}$. We fix $x_{0}$ to be $\infty$. Take a Jordan curve $L$ which encircles $b_{1}, \ldots, b_{n}$ anticlockwise and separates them from $a_{1}, \ldots, a_{n}$. We denote by $D_{+}$(resp. $\left.D_{-}\right)$the inside (resp. outside) of $L$. The problem is to find matrices $R_{+}(x)$ and $R_{-}(x)$ which are holomorphic (up to the boundary $L$ ) in $D_{+}$and $D_{-}$, respectively, such that

$$
\begin{aligned}
& R_{-}(x) Z(x)=R_{+}(x) \quad \text { if } \quad x \in L, \\
& R_{-}(x)-1=O\left(\frac{1}{x}\right) \quad \text { if } \quad|x| \rightarrow \infty .
\end{aligned}
$$

The solution $Y(x)$ to the original monodromy problem, which is normalized at $x=\infty$, is given by

$$
Y(x)= \begin{cases}R_{-}(x) Z(x) & x \in D_{-} \\ R_{+}(x) & x \in D_{+}\end{cases}
$$

The reader is referred to [8], [9] and [10] as for the standard argument below. The solution $R_{-}(x)$ should satisfy the following integral equation on the curve $L$ :

$$
R_{-}(x)-\frac{1}{2 \pi i} \int d y R_{-}(y) H(y, x)=1,
$$

where the kernel $H(y, x)$ is given by

$$
H(y, x)=\frac{Z(y)}{y} \frac{Z(x)^{-1}-1}{y} .
$$

We use the notation

$$
H\left(\begin{array}{c|c}
x_{1} \cdots x_{l} & \alpha_{1} \cdots \alpha_{l} \\
y_{1} \cdots y_{l} & \beta_{1} \cdots \beta_{l}
\end{array}\right)=\operatorname{det}\left(H\left(x_{j}, y_{k}\right)_{\alpha_{J} \beta_{k}}\right)_{j, k=1, \ldots, l} .
$$

We denote by $\Delta(\lambda)$ and $\Delta(y, x ; \lambda)$ the Fredholm determinant and the Fredholm's first minor, respectively:

$$
\Delta(\lambda)=\sum_{l=0}^{\infty} \frac{(-\lambda)^{l}}{l !} \sum_{\alpha_{1}, \ldots, \alpha_{l}=1}^{m} \int d x_{1} \cdots \int d x_{l} H\left(\begin{array}{c|c}
x_{1} \cdots x_{l} & \alpha_{1} \cdots \alpha_{l} \\
x_{1} \cdots x_{l} & \alpha_{1} \cdots \alpha_{l}
\end{array}\right) .
$$




$$
\Delta(y, x ; \lambda)_{\alpha \beta}=\sum_{l=0}^{\infty} \frac{(-\lambda)^{l}}{l !} \sum_{x_{1}, \ldots, \alpha_{l}=1}^{m} \int d x_{1} \cdots \int d x_{l} H\left(\begin{array}{c|c}
y x_{1} \cdots x_{l} & \alpha \alpha_{1} \cdots \alpha_{l} \\
x x_{1} \cdots x_{l} & \beta \alpha_{1} \cdots \alpha_{l}
\end{array}\right) .
$$

If $\Delta(1) \neq 0$ the integral equation $(6.20)$ is solvable and the solution $R_{-}(x)$ is given by

$$
R_{-}(x)=1+\int d y \frac{\Delta(y, x ; 1)}{\Delta(1)} .
$$

Note that $Z(x)$ satisfies a linear ordinary differential equation of the form

$$
\frac{d Z(x)}{d x}=\sum_{\mu=1}^{n} \sum_{j=0}^{r_{\mu}}\left(\frac{A_{\mu, j}}{\left(x-a_{\mu}\right)^{j+1}}+\frac{B_{\mu, j}}{\left(x-b_{\mu}\right)^{j+1}}\right) Z(x),
$$

with the additional condition

$$
\sum_{\mu=1}^{n} \operatorname{trace} A_{\mu, 0}=\sum_{\mu=1}^{n} \operatorname{trace} B_{\mu, 0}=0
$$

From (3.34) we have

$$
H(x, x)=\sum_{\mu=1}^{n} \sum_{j=0}^{r_{\mu}}\left(\frac{A_{\mu, j}}{\left(x-a_{\mu}\right)^{j+1}}+\frac{B_{\mu, j}}{\left(x-b_{\mu}\right)^{j+1}}\right) .
$$

Using (3.35) and (3.36) we can verify that

$$
\Delta(\lambda)=\Delta(-\lambda)
$$

Hence if $\Delta(1) \neq 0$, then we have $\Delta(-1) \neq 0$. Thus the solution $R_{-}(x)$ to the integral equation (3.33) really solves the boundary value problem (3.25) and (3.26).

We note that $\Delta(1)$, which is holomorphic if (3.24) is valid, cannot be identically zero. In fact, if $\lambda_{\alpha \beta}^{(\mu l)}=0$ and $C_{\alpha \beta}^{(\mu)}=\delta_{\alpha \beta}\left(1 \leqq \mu \leqq n, 1 \leqq \alpha, \beta \leqq m, 1 \leqq l \leqq 2 r_{\mu}\right)$, $Z(x)$ is given explicitly by

$$
Z(x)_{\alpha \beta}=\prod_{\mu=1}^{n} \varepsilon_{\alpha}^{(\mu)}(x) \delta_{\alpha \beta} .
$$

In the limit $b_{\mu}, s_{-j, \alpha}^{(\mu)}, t_{-j, \alpha}^{(\mu)} \rightarrow 0\left(1 \leqq \mu \leqq n, 0 \leqq j \leqq r_{\mu}, 1 \leqq \alpha \leqq m\right)$, we have $\Delta(1)=1$.

Thus we have proved that the solution $Y(x)$ to the monodromy problem (M1)-(M3) is meromorphic with respect to the parameters provided that (3.24) is valid. Now we shall eliminate this restriction.

Before proceeding to the general case we comment on a simpler case when the Stokes multipliers are trivial, i.e. $\lambda_{\alpha \beta}^{(\mu l)}=0\left(1 \leqq \mu \leqq n, 1 \leqq l \leqq 2 r_{\mu}, 1 \leqq \alpha, \beta \leqq m\right)$. In this case the contours of type II are absent in the Fredholm determinant 
expression for $\left\langle\varphi_{1}^{(1)} \cdots \varphi_{m}^{(n)}\right\rangle$. Hence choosing each $b_{\mu}$ closely to $a_{\mu}$ we can show (3.24). Thus we can prove the meromorphy of $Y(x)$, restricted to the submanifold where the Stokes multipliers are trivial, even at the exceptional hyperplane $\left\{t_{-r \mu, \alpha}^{(\mu)}-t_{-r \mu, \beta}^{(\mu)}=0\right\}$.

In order to treat the general case we introduce a parameter $\kappa$ and set

$$
\rho(\kappa)=\left\langle\phi_{1}^{(1)}(\kappa) \cdots \phi_{m}^{(n)}(\kappa)\right\rangle
$$

where

$$
\varphi_{\alpha}^{(\mu)}(\kappa)=\exp \left(\kappa \rho_{\alpha}^{(\mu)}\right) .
$$

If $\kappa$ is small, $\rho(\kappa) \neq 0$. Hence for some positive integer $p$ we have

$$
\frac{d^{p} \rho}{d \kappa^{p}}(1) \neq 0
$$

First let us assume that $p=1$. Then we have

$$
\left\langle\varphi_{1}^{(1)} \cdots \psi_{\alpha_{0}}^{\left(\mu_{0}\right)}\left(x_{1}\right) \psi_{\alpha_{0}}^{*\left(\mu_{0}\right)}\left(x_{2}\right) \varphi_{\alpha_{0}}^{\left(\mu_{0}\right)} \cdots \varphi_{m}^{(n)}\right\rangle \neq 0
$$

for some $\mu_{0}, \alpha_{0}$ and $x_{1}, x_{2} \in I^{\left(\mu_{0}\right)} \cup\left(\underset{\substack{l=1, \ldots, 2 r_{\mu} \\ k=1, \ldots, m-1}}{\bigcup_{k}} I I_{k}^{\left(\mu_{0} l\right)}\right)$. We set

$$
=2 \pi i\left(x-x_{0}\right) \frac{\left\langle\psi_{\alpha}^{*}\left(x_{0}\right) \varphi_{1}^{(1)} \cdots \psi_{\alpha_{0}}^{\left(\mu_{0}\right)}\left(x_{1}\right) \psi_{\alpha_{0}}^{*\left(\mu_{0}\right)}\left(x_{2}\right) \varphi_{\alpha_{0}}^{\left(\mu_{0}\right)} \cdots \varphi_{m}^{(n)} \psi_{\beta}(x)\right\rangle}{\left\langle\varphi_{1}^{(1)} \cdots \psi_{\alpha_{0}}^{\left(\mu_{0}\right)}\left(x_{1}\right) \psi_{\alpha_{0}}^{*\left(\mu_{0}\right)}\left(x_{2}\right) \varphi_{\alpha_{0}}^{\left(\mu_{0}\right)} \cdots \varphi_{m}^{(n)} \psi_{\beta}(x)\right\rangle} .
$$

We claim that $Z^{\prime}(x)$ satisfies (T1)-(T3) with additional apparent singularities (where $Z^{\prime}(x)$ is not invertible holomorphic) at $x=x_{1}$ and $x=x_{2}$. The verification of (T1), (T2) and the local behavior at $x=a_{\mu}(1 \leqq \mu \leqq n)$ are the same as for $Z(x)$.

Let us consider the local behavior of $Z^{\prime}(x)$ at $x=x_{2}$. Applying the Wick's theorem we obtain

$$
\begin{aligned}
& \left\langle\psi_{\alpha}^{*}\left(x_{0}\right) \varphi_{1}^{(1)} \cdots \psi_{\alpha_{0}}^{\left(\mu_{0}\right)}\left(x_{1}\right) \psi_{\alpha_{0}}^{*\left(\mu_{0}\right)}\left(x_{2}\right) \varphi_{\alpha_{0}}^{\left(\mu_{0}\right)} \cdots \varphi_{m}^{(n)} \psi_{\beta}(x)\right\rangle \\
& =-\sum_{l=1}^{\infty} \frac{(-)^{l}}{l !} \sum_{\mu_{1}, \ldots, \mu_{l}=1}^{n} \sum_{\alpha_{1}, \ldots, \alpha_{l}=1}^{m} \int d y_{1} \cdots \int d y_{l} \\
& \times \operatorname{det}\left[\begin{array}{ccc}
\delta_{\alpha \beta} \frac{1}{2 \pi} \frac{i}{x_{0}-x}\left\langle\psi_{\alpha}^{*}\left(x_{0}\right) \psi_{\alpha_{0}}^{\left(\mu_{0}\right)}\left(x_{1}\right)\right\rangle & \left(\left(C^{\left(\mu_{0}\right)-1}\right)_{\alpha \alpha_{k}} \frac{1}{2 \pi} \frac{i}{x-x_{k}}\right. \\
\ddots & 0 & \left.\times \varepsilon_{\alpha_{k}}^{\left(\mu_{k}\right)}\left(x_{k}\right)\right)_{k=1, \ldots, l} \\
\left\langle\psi_{\alpha_{0}}^{*\left(\mu_{0}\right)}\left(x_{2}\right) \psi_{\beta}(x)\right\rangle & \left(\left(K_{\alpha_{0} \alpha_{k}}^{\left(\mu_{0} \mu_{k}\right)}\left(x_{2}, y_{k}\right)\right)_{k=1, \ldots, l}\right. \\
\left(\frac{1}{2 \pi i} \frac{1}{x_{j}-x} \frac{1}{\varepsilon_{\alpha_{j}}^{\left(\mu_{j}\right)}\left(x_{j}\right)}\right. & \left(K_{\alpha_{j} \alpha_{0}}^{\left(\mu_{j} \mu_{0}\right)}\left(y_{j} x_{1}\right)_{j=1, \ldots, l}\left(K_{\alpha_{j} \alpha_{k}}^{\left(\mu_{j} \mu_{k}\right)}\left(y_{j}, y_{k}\right)\right)_{j, k=1, \ldots, l}\right. \\
\left.\times C_{\alpha_{j} \beta}^{\left(\mu_{j}\right)}\right)_{j=1, \ldots, l} &
\end{array}\right]
\end{aligned}
$$


If $\psi_{\alpha_{0}}^{*\left(\mu_{0}\right)}\left(x_{2}\right)=\phi_{\alpha_{0}}^{*\left(\mu_{0}\right)}\left(x_{2}\right)$, the singular part of (3.44) at $x=x_{2}$ is

$$
\begin{aligned}
& \sum_{l=0}^{\infty} \frac{(-)^{l}}{l !} \sum_{\mu_{1}, \ldots, \mu_{l}=1}^{n} \sum_{\alpha_{1}, \ldots, \alpha_{l}=1}^{m} \int d y_{1} \cdots \int d y_{l} \\
& \times \operatorname{det}\left[\begin{array}{l}
\left\langle\psi_{\alpha}^{*}\left(x_{0}\right) \psi_{\alpha_{0}}^{\left(\mu_{0}\right)}\left(x_{1}\right)\right\rangle\left(\left(C^{\left(\mu_{0}\right)-1}\right)_{\alpha \alpha_{k}} \frac{1}{2 \pi} \frac{i}{x-x_{k}} \varepsilon_{\alpha_{k}}^{\left(\mu_{k}\right)}\left(x_{k}\right)\right)_{k=1, \ldots, l} \\
\left(K_{\alpha_{j} \alpha_{0}}^{\left(\mu_{\mu_{0}}\right)}\left(y_{j}, x_{1}\right)\right)_{j=1, \ldots, l}\left(K_{\alpha_{j} \alpha_{k}}^{\left(\mu_{j} \mu_{k}\right)}\left(y_{j}, y_{k}\right)\right)_{j, k=1, \ldots, l}
\end{array}\right] \\
& \times\left(C^{\left(\mu_{0}\right)}\right)_{\alpha_{0} \beta} \frac{1}{2 \pi} \frac{i}{x_{2}-x} .
\end{aligned}
$$

Hence at $x=x_{2}$

$$
Z^{\prime}(x) C^{\left(\mu_{0}\right)-1}\left(x-x_{2}\right)^{E \alpha_{0}} \quad\left(E_{\alpha_{0}}=\left(\delta_{\alpha \alpha_{0}} \delta_{\alpha \beta}\right)_{\alpha, \beta=1, \ldots, m}\right)
$$

is holomorphic.

If $\psi_{\alpha_{0}}^{*\left(\mu_{0}\right)}\left(x_{2}\right)=\phi_{\alpha_{0}}^{*\left(\mu_{0} l\right)}\left(x_{2}\right)$ for some $\beta_{0}$ then $\lambda_{\alpha_{0} \beta 0}^{\left(\mu_{0} l\right)} \neq 0$. We define an $m \times m$ matrix $C^{\prime}$ by

$$
\left(C^{\prime-1}\right)_{\alpha \beta}=\left\{\begin{array}{cl}
1 & \alpha=\beta, \\
-\frac{\lambda_{\alpha_{0} \beta}^{\left(\mu_{0} l\right)}}{\lambda_{\alpha 0 \beta 0}^{\left(\mu_{0} l\right)}} & \alpha=\beta_{0}, \beta \neq \beta_{0}, \\
0 & \text { otherwise }
\end{array}\right.
$$

The singular part of (3.44) at $x=x_{2}$ is

$$
\begin{aligned}
& -\sum_{l=0}^{\infty} \frac{(-)^{l}}{l !} \sum_{\mu_{1}, \ldots, \mu_{l}=1}^{n} \sum_{\alpha_{1}, \ldots, \alpha_{l}=1}^{m} \int d y_{1} \ldots \int d y_{l} \\
& \times \operatorname{det}\left[\begin{array}{l}
\left.\left\langle\psi_{\alpha}^{*}\left(x_{0}\right) \psi_{\alpha_{0}}^{\left(\mu_{0}\right)}\left(x_{1}\right)\right\rangle\left(\left(C^{\left(\mu_{0}\right)-1}\right)_{\alpha \alpha_{k}} \frac{1}{2 \pi} \frac{i}{x-x_{k}} \varepsilon_{\alpha_{k}}^{\left(\mu_{k}\right)}\left(x_{k}\right)\right)_{k=1, \ldots, l}\right] \\
\left(K_{\alpha_{j} \alpha_{0}}^{\left(\mu_{j} \mu_{0}\right)}\left(y_{j}, x_{1}\right)\right)_{j=1, \ldots, m}\left(K_{\alpha_{j} \alpha_{k}}^{\left(\mu_{j} \mu_{k}\right)}\left(y_{j}, y_{k}\right)\right)_{j, k=1, \ldots, l}
\end{array}\right] \\
& \times \frac{1}{2 \pi i} \frac{1}{x_{2}-x} \frac{\varepsilon_{\alpha_{0}}^{\left(\mu_{0}\right)}(x)}{\varepsilon_{\alpha_{0}}^{\left(\mu_{0}\right)}\left(x_{2}\right)} \sum_{\beta=1}^{m} \lambda_{\alpha_{0} \beta_{0}}^{\left(\mu_{0} l\right)} C_{\beta 0}^{\left(\mu_{0}\right)} .
\end{aligned}
$$

Hence at $x=x_{2}$

$$
Z^{\prime}(x) C^{\left(\mu_{0}\right)-1} C^{\prime-1}\left(x-x_{2}\right)^{E_{\beta_{0}}}
$$

is holomorphic. Thus we have proved that at $x=x_{2}, Z^{\prime}(x)$ has an apparent singularity whose exponents of formal monodromy are zero except for single -1 .

By the same argument we can show that as a function of $x_{0}, Z^{\prime}(x)$ has an apparent singularity at $x=x_{1}$ whose exponents of formal monodromy are zero except for single -1 . Since $Z^{\prime}(x)$ is normalized so that $Z^{\prime}\left(x_{0}\right)=1$, this implies 
that at $x=x_{1}, Z^{\prime}(x)$ has an apparent singularity whose exponents are zero except for single 1 .

If $\rho \geqq 2$, by a similar argument we obtain an $m \times m$ matrix $Z^{\prime}(x)$ satisfying (T1)-(T3) with additional apparent singularities, the same number of poles and zeros.

Now we can use $Z^{\prime}(x)$ instead of $Z(x)$ in the boundary problem (3.27). We choose $L$ so that not only $b_{\mu}(1 \leqq \mu \leqq n)$ but also all the apparent singularities are inside of $L$. The final step in the proof of Theorem 1 is to show that the corresponding Fredholm determinant $\Delta^{\prime}(1)$ does not vanish identically with respect to the parameters $a_{\mu}, b_{\mu}, t_{-j, \alpha}^{(\mu)}, S_{-j, \alpha}^{(\mu)}, \lambda_{\alpha \beta}^{(\mu l)}, C_{\alpha \beta}^{(\mu)}\left(1 \leqq \mu \leqq n, 0 \leqq j \leqq r_{\mu}\right.$, $\left.1 \leqq \alpha, \beta \leqq m, 1 \leqq l \leqq 2 r_{\mu}\right)$.

Let $c_{v}, E_{\alpha v}, D^{(v)}(1 \leqq v \leqq p)$ and $c_{v},-E_{\alpha v}, D^{(v)}(p+1 \leqq v \leqq 2 p)$ be the data of additional apparent singularities. For $Z^{\prime}(x)$ constructed above the connection matrix $D^{(v)}$ at $c_{v}$ is specified by (3.46) or (3.49). We denote this special value by $D_{0}^{(v)}$. We shall show that for a generic choice of $D^{(v)}$ there exists a solution $Z^{\prime}(x)$. The solution to the twin problem is meromorphic with respect to the parameters. Thus $Z^{\prime}(x)$ is also meromorphic with respect to the parameters including $D^{(v)}$.

A little care should be taken, since the exponents at $c_{v}$ has an integer difference. At $x=c_{v}, Z^{\prime}(x)$ is of the form

$$
\begin{aligned}
& Z^{\prime}(x)=H^{(v)} \hat{Z}^{\prime(v)}(x)\left(x-c_{v}\right)^{ \pm E_{\alpha_{v}} D^{(v)},} \\
& \hat{Z}^{\prime(v)}(x)=1+\sum_{j=1}^{\infty} Z_{j}^{\prime(v)}\left(x-c_{v}\right)^{j} .
\end{aligned}
$$

We define $B_{j}^{(v)}(j \leqq 0)$ by

$$
\frac{d Z^{\prime}(x)}{d x}=H^{(v)}\left(\frac{ \pm E_{\alpha_{v}}}{x-c_{v}}+\sum_{j=0}^{\infty} B_{j}^{(v)}\left(x-c_{v}\right)^{j}\right) H^{(v)-1} Z^{\prime}(x)
$$

We have the constraints

$$
\begin{array}{ll}
\left(B_{0}^{(v)}\right)_{\alpha_{\nu} \beta}=0 & \left(1 \leqq v \leqq p, \alpha_{\nu} \neq \beta\right), \\
\left(B_{0}^{(v)}\right)_{\beta \alpha_{\nu}}=0 & \left(p+1 \leqq \nu \leqq 2 p, \alpha_{v} \neq \beta\right) .
\end{array}
$$

On the other hand, if we solve (3.51) assuming (3.52), $\left(Z_{j}^{\prime(v)}\right)_{\alpha_{v} \beta}\left(1 \leqq v \leqq p, \alpha_{v} \neq \beta\right)$ and $\left(Z_{j}^{\prime}(v)\right)_{\beta \alpha_{v}}\left(p+1 \leqq v \leqq 2 p, \alpha_{\nu} \neq \beta\right)$ are undetermined.

Let us consider the analytic space of singular data $\mathscr{N}^{\prime}$ consisting of those for the twin problem, $H^{(v)}(1 \leqq v \leqq 2 p),\left(Z_{1}^{\prime(v)}\right)_{\alpha_{v} \beta}(1 \leqq v \leqq p)$ and $\left(Z_{1}^{\prime(v)}\right)_{\beta \alpha_{\nu}}(p+1$ $\leqq v \leqq 2 p$ ), which are subject to the constraints (3.52). We also consider the 
analytic space $\mathscr{M}^{\prime}$ of monodromy data consisting of those for the twin problem and $D^{(v)}(1 \leqq v \leqq 2 p)$. The map from $\mathscr{N}^{\prime}$ to $\mathscr{M}^{\prime}$ which maps a singular data to the corresponding monodromy data is analytic and one to one. Hence the intersection of the image of $\mathscr{N}^{\prime}$ and the fiber which contains the point $\left(a_{\mu}\right.$, $b_{\mu}, t_{-j, \alpha}^{(\mu)}, s_{-j, \alpha}^{(\mu)}, \lambda_{\alpha \beta}^{(\mu l)}, C_{\alpha \beta}^{(\mu)}\left(1 \leqq \mu \leqq n, 0 \leqq j \leqq r_{\mu}, 1 \leqq \alpha, \beta \leqq m, 1 \leqq l \leqq 2 r_{\mu}\right), D_{0}^{(v)}$ $(1 \leqq v \leqq 2 p))$ is dense in the latter.

Now let us continue $Z^{\prime}(x)$ with respect to $t_{-j, \alpha}^{(\mu)}, s_{-j, \alpha}^{(\mu)}$ and $\lambda_{\alpha \beta}^{(\mu)}(1 \leqq \mu \leqq n$, $\left.0 \leqq j \leqq r_{\mu}, 1 \leqq \alpha, \beta \leqq m\right)$. If they are small enough, Theorem 2.3 of [2] assures that we can solve the monodromy problem $\mathscr{P}$ with the data $a_{\mu}, t_{-j, \alpha}^{(\mu)}, \lambda_{\alpha, \beta}^{(\mu l)}, C_{\alpha \beta}^{(\mu)}$ as well as $\mathscr{P}^{\prime \prime}$ with the data $b_{\mu}, s_{-j, \alpha}^{(\mu)}, \lambda_{\alpha \beta}^{(\mu l)}, C_{\alpha \beta}^{(\mu)}$. Then for a generic choice of $D^{(v)}$ the monodromy problem $\mathscr{P}^{\prime}$ with the data $b_{\mu}, s_{-j, \alpha}^{(\mu)}, \lambda_{\alpha \beta}^{(\mu l)}, C_{\alpha \beta}^{(\mu)}$ and $c_{v}, \pm E_{\alpha_{v}}$, $D^{(v)}$ admits a solution. Hence by the lemma in Appendix we know that an analytic continuation of $Z^{\prime}(x)$ can be identified with the solution $Y\left(x_{0}, x ; \mathscr{P}_{t}+\mathscr{P}^{\prime}\right)$ to the composite problem $\mathscr{P}_{t}+\mathscr{P}^{\prime}$. Then considering the limit $t \rightarrow 0$, we can show that $\Delta^{\prime}(1)$ does not vanish identically. Thus we have proved Theorem 1.

\section{§4. Proof of Theorem 2}

First we shall show that the $\tau$ function for the twin problem is analytic except at the fixed singularities. The asymptotic series for the solution $Z(x)$ to the twin problem is of the form

$$
Z(x) \sim\left\{\begin{array}{lll}
G_{+}^{(\mu)} \hat{Z}_{+}^{(\mu)}(x) e^{S^{(\mu)}(x)} C^{(\mu)} & \text { at } & x=a_{\mu} \\
G_{-}^{(\mu)} \hat{Z}_{-}^{(\mu)}(x) e^{S^{(\mu)}(x)} C^{(\mu)} & \text { at } & x=b_{\mu},
\end{array}\right.
$$

where $S^{(\mu)}(x)$ is an $m \times m$ diagonal matrix with

$$
\begin{aligned}
S^{(\mu)}(x)_{\alpha \alpha}= & \sum_{j=1}^{r_{\mu}}\left(t_{-j, \alpha}^{(\mu)} \frac{\left(x-a_{\mu}\right)^{-j}}{-j}+t_{0, \alpha}^{(\mu)} \log \left(x-a_{\mu}\right)\right. \\
& \left.-S_{-j, \alpha}^{(\mu)} \frac{\left(x-b_{\mu}\right)^{-j}}{-j}-t_{0, \alpha}^{(\mu)} \log \left(x-b_{\mu}\right)\right)
\end{aligned}
$$

Set

$$
\begin{aligned}
\omega^{\prime}= & -\sum_{\mu=1}^{n} \operatorname{Res}_{x=a_{\mu}} \operatorname{trace} \hat{Z}_{+}^{(\mu)}(x)^{-1} \frac{\partial \hat{Z}_{+}^{(\mu)}(x)}{\partial x} d_{t} S^{(\mu)}(x) \\
& -\sum_{\mu=1}^{n} \operatorname{Res}_{x=b_{\mu}} \operatorname{trace} \hat{Z}_{-}^{(\mu)}(x)^{-1} \frac{\partial \hat{Z}_{-}^{(\mu)}(x)}{\partial x} d_{t} S^{(\mu)}(x) .
\end{aligned}
$$

Then by the same argument as in Section 5 of [2] we can show that 


$$
\omega^{\prime}=d \log \left\langle\varphi_{1}^{(1)} \cdots \varphi_{m}^{(n)}\right\rangle .
$$

The difference between the 1-form $\omega_{\text {twin }}$ of the definition in [1] for the twin problem and $\omega^{\prime}$ defined above causes the following discrepancy between the $\tau$ function $\tau_{\text {twin }}$ for the twin problem and $\left\langle\varphi_{1}^{(1)} \ldots \varphi_{m}^{(n)}\right\rangle$ :

$$
\tau_{\mathrm{twin}}=\left\langle\varphi_{1}^{(1)} \cdots \varphi_{m}^{(n)}\right\rangle \prod_{\mu=1}^{n} \prod_{\alpha=1}^{m} \tau_{\alpha}^{(\mu)}
$$

where $\tau_{\alpha}^{(\mu)}$ is the $\tau$ function for the monodromy problem whose solution is $\varepsilon_{\alpha}^{(\mu)}(x)$. Thus $\tau_{\text {twin }}$ is analytic except at the fixed singularities. Hence the one form $\omega_{\text {twin }}$ for the twin problem has only simple poles with residues of positive integers.

Now we denote by $\mathscr{P}^{\prime}$ (resp. $\mathscr{P}_{t}$ ) the monodromy problem with the data $a_{\mu}, t_{-j, \alpha}^{(\mu)}, \lambda_{\alpha \beta}^{(\mu l)}, C_{\alpha \beta}^{(\mu)}$ (resp. $\left.t b_{\mu}, t^{j} t_{-j, \alpha}^{(\mu)}, \lambda_{\alpha \beta}^{(\mu l)}, C_{\alpha \beta}^{(\mu)}\right)$. We shall use the notation in Appendix. The asymptotic series at $a_{\mu}$ for $Y\left(x_{0}, x ; \mathscr{P}_{t}+\mathscr{P}^{\prime}\right)$ reads

$$
Y\left(x_{0}, x ; \mathscr{P}_{t}+\mathscr{P}^{\prime}\right) \sim G^{(\mu)}(t) \hat{Z}^{(\mu)}(x, t) e^{T^{(\mu)}(x)} C^{(\mu)} .
$$

Then the poles of the meromorphic 1-form

$$
\omega(t)=-\sum_{\mu=1}^{n} \operatorname{Res}_{x=a_{\mu}} \operatorname{trace} \hat{Z}^{(\mu)}(x, t)^{-1} \frac{\partial \hat{Z}^{(\mu)}(x, t)}{\partial x} d_{t} T^{(\mu)}(x)
$$

are simple and their residues are positive integers.

Theorem 1 assures that the closed 1-form $\omega_{\mathscr{P}}$, for $\mathscr{P}^{\prime}$ is meromorphic. Let us prove that the poles of $\omega_{\mathscr{P}^{\prime}}$ are simple and their residues are positive integers. If $\left\{a_{\mu}, t_{-j, \alpha,\}_{1} \leqq \mu \leqq n, 1 \leqq j \leqq r_{\mu}, 1 \leqq \alpha \leqq m}\right.$ is not contained in the pole locus, then $Y\left(x_{0}, x\right.$, $\left.\mathscr{P}^{\prime}\right)$ exists. Then, for a generic choice of $b_{\mu}$ and $s_{-j, \alpha}^{(\mu)}, Y\left(x_{0}, x, \mathscr{P}_{t}\right)$ also exists. Then the lemma in Appendix implies

$$
\lim _{t \rightarrow 0} \omega(t)=\omega_{\mathscr{P}^{\prime}} .
$$

Because the residues of $\omega(t)$ are constant, $\omega_{\mathscr{P}^{\prime}}$ has only simple poles. Finally, since the residues of $\omega(t)$ are positive integers, so are the residues of $\omega_{\mathscr{P}}$. Thus we have proved Theorem 2.

\section{Appendix}

Let us consider two different monodromy problems $\mathscr{P}_{t}(t>0)$ and $\mathscr{P}^{\prime}$ with the following monodromy data:

$$
\mathscr{P}_{t}: t a_{\mu}, t^{j} t_{-j, \alpha}^{(\mu)}, \lambda_{\alpha \beta}^{(\mu l)}, C_{\alpha \beta}^{(\mu)}\left(1 \leqq \mu \leqq n, 0 \leqq j \leqq r_{\mu}, 1 \leqq l \leqq 2 r_{\mu}, 1 \leqq \alpha, \beta \leqq m\right),
$$


$\mathscr{P}^{\prime}: a_{\mu}^{\prime}, t_{-j, \alpha}^{(\mu)^{\prime}}, \lambda_{\alpha \beta}^{(\mu l))^{\prime}}, C_{\alpha \beta}^{(\mu)^{\prime}}\left(1 \leqq \mu \leqq n^{\prime}, 0 \leqq j \leqq r_{\mu}^{\prime}, 1 \leqq l \leqq 2 r_{\mu}^{\prime}, 1 \leqq \alpha, \beta \leqq m\right)$.

We take Jordan curves $C_{t}$ and $C^{\prime}$ with anticlockwise orientation which encircles $t a_{1}, \ldots, t a_{n}$ and $a_{1}^{\prime}, \ldots, a_{n^{\prime}}^{\prime}$, respectively. We assume that the regions surrounded by $C_{t}$ and $C^{\prime}$ are disjoint, and that $0 \in C$ is not contained in $C^{\prime}$.

We denote by $Y\left(x_{0}, x ; \mathscr{P}_{t}\right)$ (resp. $\left.Y\left(x_{0}, x ; \mathscr{P}^{\prime}\right)\right)$ the solution to the monodromy problem $\mathscr{P}_{t}$ (resp. $\left.\mathscr{P}^{\prime}\right)$. Similarly we denote by $Y\left(x_{0}, x ; \mathscr{P}_{t}+\mathscr{P}^{\prime}\right)$ the solution to the composite problem $\mathscr{P}_{t}+\mathscr{P}^{\prime}$ whose data are the union of those of $\mathscr{P}_{t}$ and $\mathscr{P}^{\prime}$.

Lemma. Assume that $Y\left(x_{0}, x ; \mathscr{P}_{1}\right)$ and $Y\left(x_{0}, x ; \mathscr{P}^{\prime}\right)$ exist. Then for small $t Y\left(x_{0}, x, \mathscr{P}_{t}+\mathscr{P}^{\prime}\right)$ exists and the following limit is locally uniform:

$$
\lim _{t \rightarrow 0} Y\left(x_{0}, x ; \mathscr{P}_{t}+\mathscr{P}^{\prime}\right)=Y\left(x_{0}, x ; \mathscr{P}^{\prime}\right) .
$$

Before proceeding to the proof we prepare the following. Let $Y(x)$ be any solution to the monodromy problem (M2) and (M3). Using free fermion fields $\psi_{\alpha}^{*}(x), \psi_{x}(x)(1 \leqq \alpha \leqq m)$ satisfying (3.8), we set

$$
\varphi=: \exp \left\{\iint d x d x^{\prime} \sum_{\alpha, \beta=1}^{m} \frac{1}{2 \pi i} \frac{1}{x-x^{\prime}}\left(Y(x)^{-1} Y\left(x^{\prime}\right)\right)_{\alpha \beta} \psi_{\alpha}(x) \psi_{\beta}^{*}\left(x^{\prime}\right)\right\}
$$

The integration contours for $x$ and $x^{\prime}$ in (A2) are Jordan curve $C$ which encircles all the singularities anticlockwise. When $x$ and $x^{\prime}$ coincides with each other we deform the contour for $x^{\prime}$ outwardly. If we take $x_{0}$ and $x$ outside of $C$, we have

$$
2 \pi i\left(x-x_{0}\right)\left\langle\psi_{\alpha}^{*}\left(x_{0}\right) \varphi \psi_{\beta}(x)\right\rangle=\left(Y\left(x_{0}\right)^{-1} Y(x)\right)_{\alpha \beta} .
$$

We denote by $\varphi_{t}$ (resp. $\varphi^{\prime}$ ) the Clifford operator defined by (A2) with $Y(x)$ replaced by $Y\left(\infty, x ; \mathscr{P}_{t}\right)$ (resp. $Y\left(\infty, x ; \mathscr{P}^{\prime}\right)$ ). Then if $\left\langle\varphi_{t} \varphi^{\prime}\right\rangle \neq 0$ the solution $Y\left(x_{0}, x ; \mathscr{P}_{t}+\mathscr{P}^{\prime}\right)$ is given by

$$
Y\left(x_{0}, x ; \mathscr{P}_{t}+\mathscr{P}^{\prime}\right)=2 \pi i\left(x-x_{0}\right) \frac{\left\langle\psi_{\alpha}^{*}\left(x_{0}\right) \varphi_{t} \varphi^{\prime} \psi_{\beta}(x)\right\rangle}{\left\langle\varphi_{t} \varphi^{\prime}\right\rangle} .
$$

Since we have

$$
Y\left(\infty, x, \mathscr{P}_{t}\right)=Y\left(\infty, \frac{x}{t}, \mathscr{P}_{1}\right)
$$

in the limit $t \rightarrow 0$ we have

$$
\lim _{t \rightarrow 0} \varphi_{t}=: \exp \iint d x d x^{\prime} \sum_{\alpha=1}^{m} \frac{1}{2 \pi i} \frac{1}{x-x^{\prime}} \psi_{\alpha}(x) \psi_{\alpha}^{*}\left(x^{\prime}\right): .
$$


Using (A6) we have

$$
\begin{aligned}
& \lim _{t \rightarrow 0}\left\langle\psi_{\alpha}^{*}\left(x_{0}\right) \varphi_{t} \varphi^{\prime} \psi_{\beta}(x)\right\rangle=\left\langle\psi_{\alpha}^{*}\left(x_{0}\right) \varphi^{\prime} \psi_{\beta}(x)\right\rangle, \\
& \lim _{t \rightarrow 0}\left\langle\varphi_{t} \varphi^{\prime}\right\rangle=1 .
\end{aligned}
$$

The latter implies that for small $t$

$$
\left\langle\varphi_{t} \varphi^{\prime}\right\rangle \neq 0 .
$$

Hence (A3) and (A4) prove the lemma.

\section{References}

[1] Jimbo, M., Miwa, T. and Ueno, K., Physica, 2D (1981), 306.

[2] Miwa, T., Publ. RIMS, Kyoto Univ., 17 (1981), 665.

[3] Ablowitz, M. J., Ramani, A. and Segur, H., Lett. Nuovo Cimento, 23 (1978), 333.

[4] Okamoto, K., Proc. Japan Acad., 56A (1980), 264, 367.

[5] Jimbo, M. and Miwa, T., Monodromy preserving deformation of linear ordinary differential equations with rational coefficients, II, Physica, 2D (1981), 407.

[6] Garnier, R., Ann. Ec. Nornt. Sup., 29 (1912), 1.

[7] Oishi, S., J. Phys. Soc. Japan, 49 (1980), 1647.

[ 8 ] Hilbert, D., Verh. des 3. internat. Math. Kongr. Heidelberg, (1904), 233.

[9] Saito, T., Sugaku, 12 (1960), 145 (in Japanese).

[10] Plemelj, J., Problems in the sense of Riemann and Klein, Interscience, 1960.

[11] Birkhoff, G. D., Proc. Amer. Acad., 49 (1913), 521.

[12] Sato, M., Miwa, T. and Jimbo, M., Publ. RIMS, Kyoto Univ., 14 (1978), 223; 15 (1979), 201, 577, 871; 16 (1980), 531.

[13] Ueno, K., RIMS, Kokyuroku, 388 (1980), 102 (in Japanese).

[14] Wu, T. T., McCoy, B. M., Tracy, C. A. and Barouch, E., Phys. Rev., B13 (1976), 316.

[15] Sato, M., Miwa, T. and Jimbo, M., Proc. Japan Acad., 53A (1977), 147, 153, 183.

[16] Jimbo, M., Miwa, T., Môri, Y. and Sato, M., Physica, $\mathbb{1 D}$ (1980), 80.

[17] Jimbo, M. and Miwa, T., Proc. Japan Acad., 56A (1980), 143, 149, 269.

Added in Proof: By a letter from Professor B. Malgrange I learned that he also proved the Painlevé property for the Schlesinger equation. 
